\title{
Identification, discrimination, and selective adaptation of simultaneous musical intervals
}

\author{
ROBERT J. ZATORRE \\ Brown University, Providence, Rhode Island 02912 \\ and \\ ANDREA R. HALPERN \\ Brandeis University, Waltham, Massachusetts 02154
}

\begin{abstract}
Four experiments investigated perception of major and minor thirds whose component tones were sounded simultaneously. Effects akin to categorical perception of speech sounds were found. In the first experiment, musicians demonstrated relatively sharp category boundaries in identification and peaks near the boundary in discrimination tasks of an interval continuum where the bottom note was always an $F$ and the top note varied from $A$ to $A$ flat in seven equal logarithmic steps. Nonmusicians showed these effects only to a small extent. The musicians showed higher than predicted discrimination performance overall, and reaction time increases at category boundaries. In the second experiment, musicians failed to consistently identify or discriminate thirds which varied in absolute pitch, but retained the proper interval ratio. In the last two experiments, using selective adaptation, consistent shifts were found in both identification and discrimination, similar to those found in speech experiments. Manipulations of adapting and test showed that the mechanism underlying the effect appears to be centrally mediated and confined to a frequency-specific level. A multistage model of interval perception, where the first stages deal only with specific pitches may account for the results.
\end{abstract}

Categorical perception is said to occur when signals that vary continuously are assigned to a few discrete categories by a perceiver. In the most extreme case of categorical perception, discrimination is limited by identification, in contrast to more common psychophysical principles which indicate that many more stimuli can be discriminated than can be identified on an absolute basis (Miller, 1956). Typically, the occurrence of categorical perception has been operationally defined by (1) the presence of distinct labeling categories separated by a sharp boundary, (2) peaks in discrimination near the boundary, with low performance within categories, and (3) a close correspondence between obtained discrimination and that predicted by the hypothesis that dis-

We wish to express our thanks and appreciation to Dr. Peter D. Eimas for his support and highly valuable advice throughout our work. Helpful comments were also provided by Drs. A. Wingfield, S. Blumstein, and W. F. Ganong. We also wish to thank Dr. A. M. Liberman for his permission to use the facilities at the Haskins Laboratories. This research was supported in part by NICHD Grant HD 05331-09 to P. D. Eimas. Some of the results of these experiments were presented at the 97 th meeting of the Acoustical Society of America, Cambridge, Massachusetts, June 1979. Andrea R. Halpern is currently at the Psychology Department, Stanford University, Stanford, California 94305. Reprint requests and correspondence may be sent to Robert J. Zatorre, Psychology Department, Brown University, Providence, Rhode Island 02912. crimination can occur only insofar as stimuli can be identified as different (Studdert-Kennedy, Liberman, Harris, \& Cooper, 1970). When first investigated, this form of perception was presumed to operate only in the decoding of consonants in speech (Liberman, Harris, Hoffman, \& Griffith, 1957). Nonspeech sounds, vowels, and speech cues such as formant transitions in nonspeech contexts failed to elicit categorical perception (Eimas, 1963; Liberman, Harris, Kinney, \& Lane, 1961; Mattingly, Liberman, Syrdal, \& Halwes, 1971).

Subsequent investigations have modified original claims that speech perception involves only phonetic (i.e., categorical) information and that speech is the only mode perceived categorically. An auditory (i.e., noncategorical) level of processing can be discerned by the use of reaction times which show increasing latencies when there is uncertainty as to category membership, especially as the signal approaches a phonetic boundary (Studdert-Kennedy, Liberman, \& Stevens, 1963; Pisoni \& Tash, 1974). In addition, processing of the speech sounds can be interrupted by interfering with short-term memory stores which are thought to be auditory in nature (Pisoni, 1973, 1975; Fujisaki \& Kawashima, Note 1).

Use of selective adaptation has also implicated auditory processing in addition to phonetic process- 
ing in speech perception. Repeated presentation of an auditory stimulus can shift the locus of the phonetic boundary presumably because specific detectors have been fatigued or adapted (Eimas \& Corbit, 1973; Eimas, Cooper, \& Corbit, 1973). Adaptors that share either phonetic (Diehl, 1975) or acoustic (Sawusch, 1977) characteristics with the testing stimuli have produced adaptation effects, though most investigators agree that the results can be best explained in purely auditory terms (cf. Eimas \& Miller, 1978 , for a review).

The claim that speech is not the only mode in which categorical perception occurs is substantiated by demonstrating categorical perception for nonlinguistic stimuli, such as rise time of sawtooth waves (Cutting \& Rosner, 1974; Cutting, Rosner, \& Foard, 1976), temporal order of auditory stimuli (Miller, Wier, Pastore, Kelly, \& Dooling, 1976; Pisoni, 1977), rhythmic units (Raz \& Brandt, 1977), and flicker frequency of a light (Pastore, Ahroon, Baffuto, Friedman, Puleo, \& Fink, 1977). These results imply that categorical perception may be a much more general phenomenon than originally thought. It may occur, therefore, whenever the physical stimulus structure is not stored in memory, the information instead being reduced to a few discrete categories. Thus, the mechanism underlying categorical perception may be useful for dealing with the memory demands involved in the rapid decoding of complex information, such as must occur in speech perception and also perhaps in some aspects of music perception.

In the case of musical stimuli, the relevant cues are the pitches of pure tones and the frequency ratios between tones. These acoustic cues are not relevant to phonemic distinctions, unlike, for instance, the dimension of rise time which cues the fricativeaffricate distinction. Furthermore, musical intervals are particularly useful for experimentation due to their extremely simple acoustic nature. Another reason for studying musical interval perception is that the role of experience in the development of one instance of categorical perception can be investigated, since the degree of musical training can be controlled in the subject population.

Locke and Kellar (1973) constructed a continuum of three-note chords where the central note varied in small steps. They found categorical perception by trained musicians but continuous perception by nonmusicians. However, there were some problems with this study, particularly with regard to their signal detection analyses (Pastore, 1976). Blechner (Note 2) found similar results using simultaneous two-note intervals, as did Siegel and Siegel (1977a, 1977b), who used sequential two-note intervals. In these studies, musically trained subjects tended to classify acoustically ambiguous chords as "major," "minor," "diminished," etc., in much the same ways as listeners classify ambiguous speech signals as one phoneme or another. Burns and Ward (1978) extended the work on perception of sequential intervals by constructing continua of stimulus intervals whose bottom note varied randomly within a certain range. The top note, however, was always kept in the proper frequency relationship for the interval being tested. Burns and Ward obtained discrimination functions very close to those predicted on the basis of the identification data (i.e., under the assumption that stimuli can be discriminated only insofar as they can be labeled as different). Burns, Carney, and Ward (1976) have presented preliminary data on selective adaptation of sequential intervals. Based on a single subject, they reported boundary shifts when endpoint stimuli were used as adaptors. Adaptation seemed also to occur in spite of the fact that the absolute pitch of the intervals varied within a small range; this would imply that the adaptation effect cannot be completely auditory in origin. However, these preliminary data do not allow a precise estimate of the nature of the effect or its locus.

To summarize: (1) Research with musical intervals seems to indicate that categorical perception is not unique to speech or to speech-relevant acoustic cues. (2) There is the possibility that musical interval categories are learned, as the degree of categorical perception shown by the subjects seems to vary with the amount of musical training, and also because nonWestern cultures use different musical interval systems.

This series of studies was designed to explore the perception of simultaneous musical intervals by musicians and nonmusicians, paralleling work in speech perception. We hoped to show that analogous processes operate for speech and music perception, thereby demonstrating that categorical perception is a relatively general phenomenon. The use of simultaneous (as opposed to sequential) intervals as stimuli is reasonable; based on the principles of Western music theory, an interval is defined as the frequency ratio between two tones. Simultaneous and sequential intervals correspond musically to harmonic and melodic intervals, respectively.

Experiment 1 required identification and discrimination of eight simultaneous intervals ranging in frequency ratio between a major and minor third. Reaction times to both tasks were also recorded. Experiment 2 used the same tasks as Experiment 1, but employed stimuli whose absolute frequency varied within a small range in order to investigate how abstract (i.e., how independent of the specific component pitches) the musical categories were. Experiment 3 applied selective adaptation with one endpoint of the stimulus continuum to establish validity of the technique for musical interval perception. Experiment 4 varied the adaptor and test stimuli in several conditions to begin pinpointing the contribution of auditory vs. more abstract ("phonetic") and central vs. peripheral ${ }^{t}$ components to the adaptation effect. 


\section{EXPERIMENT 1}

This experiment was designed to show to what extent musicians and nonmusicians categorically perceive simultaneous musical intervals.

\section{Method}

Subjects. A total of 16 Brandeis University graduate and undergraduate students served as subjects. The musicians, one undergraduate and seven graduate music students, were students of composition, music history, or musicology. However, all were proficient (at least 8 years of training) on at least one instrument. Pianists, brass, woodwind, and string players were represented by at least one subject. No subjects reported possessing absolute pitch, but all had studied music theory and ear training. The nonmusicians were eight undergraduates who had very little or no performing experience and had never studied music formally. No subject reported any speech or hearing disorders.

Stimulus materials. Stimuli consisted of two simultaneously presented pure tones. The lower tone was always an F $(349 \mathrm{~Hz})$, and the higher tone ranged from $A$ flat $(415 \mathrm{~Hz})$, which forms an interval of 300 cents (a minor third), to A natural $(440 \mathrm{~Hz})$, which forms an interval of 400 cents (a major third). The top notes varied from 415 to $440 \mathrm{~Hz}$ in seven logarithmically equal stops (14.29 cents each) to form a continuum of eight intervals. The smallest step was $3.4 \mathrm{~Hz}$ and the largest was $3.6 \mathrm{~Hz}$.

Pure tones were generated on a Hewlett-Packard 207A audio sweep oscillator. Fine control of frequency was obtained by monitoring the output of the oscillator on a Hewlett-Packard 5212A electric digital counter. Tones were fed into the pulse code modulation system (Cooper \& Mattingly, 1969) at the Haskins Laboratories, where they were digitized, truncated to a duration of $500 \mathrm{msec}$ with instantaneous rise and fall times, and combined into the simultaneous intervals. The stimuli were converted back to analog form and recorded onto magnetic tape.

Two separate random-order identification tapes were made. For each tape, 15 examples of each of the 8 stimuli were randomized for a total of 120 stimuli. Each stimulus was followed by a 3-sec response period.

Two discrimination tapes were also prepared. Each stimulus was paired with the stimulus two steps (approximately $7 \mathrm{~Hz}$ ) away on the continuum to form pairs such as Stimuli 1 and 3,2 and 4, etc. Likewise, each stimulus was paired with the stimulus three steps (approximately $10 \mathrm{~Hz}$ ) away on the continuum. ${ }^{2}$ One oddity triad consisted of two presentations of one member of the pair, and a third presentation of the other member, all separated by $1 \mathrm{sec}$ of silence. Each oddity triad was followed by a 3-sec response period. The odd stimulus appeared equally often as the first, second, or third interval of the triad. Each triad appeared 12 times per tape for a total of 132 trials. Order of presentation of the triads was randomized.

Procedure. Tapes were played on a Teac 3300 stereo tape recorder, whose output was fed through a Scott Stereomaster $299 \mathrm{~F}$ amplifier to both channels of good-quality headphones for binaural presentation. Intensity of the stimuli was maintained at approximately $73 \mathrm{~dB}$ SPL.

In order to familiarize the subjects with the stimuli and task, they first heard examples of the continuum endpoints. For musicians, these were labeled as major or minor thirds; for nonmusicians, they were called " 1 " and " 2 ," respectively. The labels were provided by the experimenter in both cases. Nonmusicians were allowed to hear the endpoint stimuli as often as they wished throughout the experiment.

The identification task required subjects to decide whether each of the 120 stimuli sounded closer to a minor or to a major third ("1" or " 2 " for nonmusicians). In the discrimination task, the subjects were instructed to respond verbally " 1 ," "2," or " 3 " to indicate the position in the set of the odd member. Guessing was encouraged in both tasks. The responses were recorded on magnetic tape in order to analyze the reaction times, which were measured by using a voice-triggered storage oscilloscope. Accuracy was obtained to the nearest $12 \mathrm{msec}$.

There were two sessions of approximately $40 \mathrm{~min}$ each, and at least 3 days intervened between testing sessions. Order of task presentation was counterbalanced across subjects. All subjects were run individually.

\section{Results and Discussion}

Mean percentage of each stimulus labeled as minor by all eight musicians is shown in Figure 1. The identification function shows distinct categories for major and minor thirds, i.e., Stimuli 1, 2, and 3 are clearly considered minor; 7 and 8 are classified as major. The region of transition between the two categories is sharp and distinct. Each data point is based on a total of 240 trials.

Reaction times for identification of each stimulus are also shown in Figure 1. Reaction times increase for the stimuli in the middle of the continuum $[F(5,35)$ $=43.92, p<.001$, for the main effect of stimulus pair]; this indicates that reaction time, and presumably decision time, is slowest where there exists maximum uncertainty about the identity of the stimulus. By a Neuman-Keuls test, reaction times to Stimuli 1, 2, and 3 failed to show a difference: responses to 7 and 8 were also not different. Reaction times only increase where there is a change in certainty reflected by the identification function. Not only was, for example, Stimulus 3 being called minor $100 \%$ of the time, but also the subjects were as quick to call it minor as they were for Stimuli 1 and 2, even though Stimulus 3 was physically on the sharp (higher frequency) side of minor.

Mean percentage correct for obtained and predicted two-step discrimination as a function of stimulus pair is shown in Figure 2. Three-step functions are not presented, since a ceiling effect occurred. The obtained discrimination function (dashed line) clearly shows an increase in performance which peaks at the category boundary. This observation was confirmed by a one-way analysis of variance, which shows a significant effect of stimulus pair $[F(5,35)=9.58, p<$ .001 ]. Each data point is based on 192 trials.

The dotted line in Figure 2 is the discrimination function predicted if discrimination were strictly limited by identification. ${ }^{3}$ Musicians clearly performed above the predicted level, and this was confirmed in a two-way analysis of variance which showed an effect of obtained vs. predicted functions $[F(1,7)=57.25, \mathrm{p}<.001]$.

An important characteristic shown by experiments which demonstrate categorical perception is a good correspondence of peak performance between obtained and predicted functions. If a phonetic or symbolic level of analysis is being employed, then best performance should occur when the two stimuli being discriminated are farthest from each other on either side of the category boundary. That pattern of results is shown here, where the two functions have 


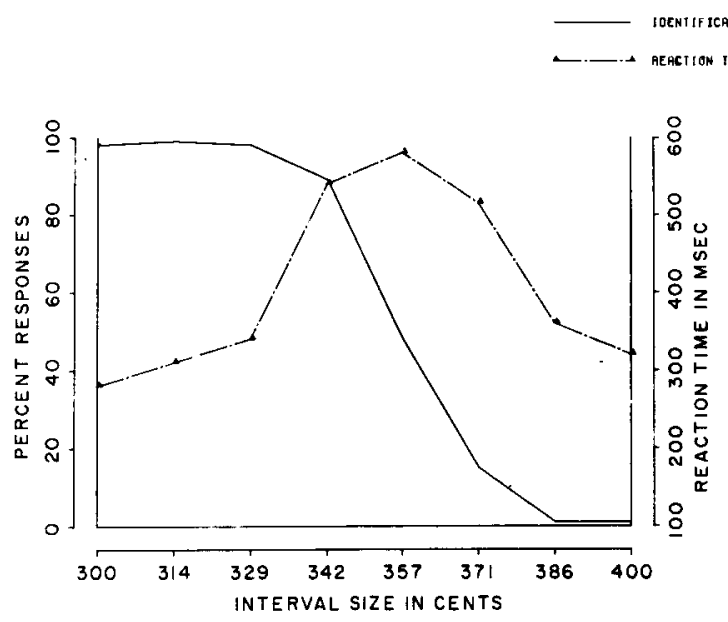

Figure 1. Mean percent identification responses for eight musicians from Experiment 1 (read against left axis), and mean reaction time for identification for the same subjects (read against right axis).

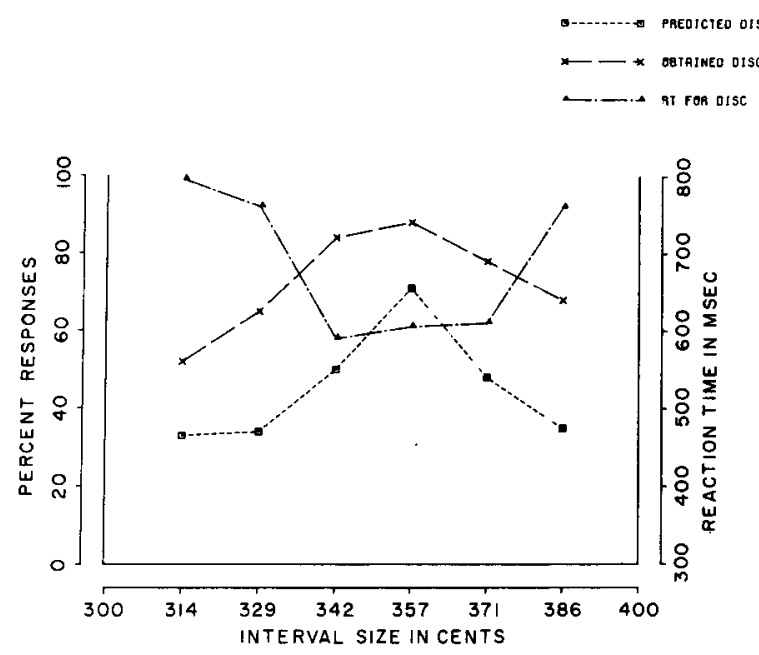

Figure 2. Mean percent correct predicted and obtained discrimination scores for all eight musicians from Experiment 1 (read against left axis), and mean reaction time for discrimination in the same subjects (read against right axis; note shift in scale with respect to Figure 1).

essentially the same shape; the peak performance is at the same stimulus pair as predicted, and there is no significant Obtained vs. Predicted by Stimulus Pair interaction $[F(1,35)=2.37, \mathrm{p}>.10]$. This latter result indicates that the hypothesized auditory information is available at all stimulus pair comparisons, and increases performance by a constant amount. Since this increase seems to be independent of boundary effects, it is reasonable to propose that it arises from a precategorical processing stage.

Mean reaction times to the discrimination task for all eight musicians are also shown in Figure 2. As shown, reaction times change as a function of stimulus pair $[\mathrm{F}(5,35)=2.59, \mathrm{p}<.05]$. The fastest times are those for stimulus pairs which are near or straddle the perceptual boundary as determined by the identification function in Figure 1. Reaction times are highest where comparisons involve pair members which lie within the same symbolic categories. Quick reaction times also correspond to best performance on the task. Note that the reaction time scales on the right-hand ordinates of Figures 1 and 2 are slightly displaced with respect to each other. It is interesting that the longest reaction times for identification, near the boundary, correspond very well to the shortest reaction times for discrimination, again near the boundary. This pattern implies that pairs from different categories require only a decision on the symbolic level (i.e., "major" or "minor") for discrimination. When this decision cannot be made on that basis alone, then an auditory level of processing increases reaction time for same-category members. Conversely, in identification, reaction times are quickest for the "best" (i.e., closest to the endpoint) stimuli. When the stimulus does not match the endpoints, then auditory information must be called upon, again increasing reaction times (cf. Pisoni, 1973).

Mean percentage of times that each stimulus was labeled " 2 " (minor) by all eight nonmusicians is shown in Figure 3. The identification function was not as sharp as that for the musicians, indicating less consistent use of category labels, but the basic shape of the function was similar to the musicians'. Mean percent correct two-step discrimination is also shown in Figure 3: discrimination performance by nonmusicians showed a flattened curve as compared with the musicians. There nevertheless remained a statistically reliable peak between Stimuli 4 and $6[F(5,35)=$ $3.43, \mathrm{p}<.05$ ], just as with the musicians. Obtained performance was no better than predicted, however,
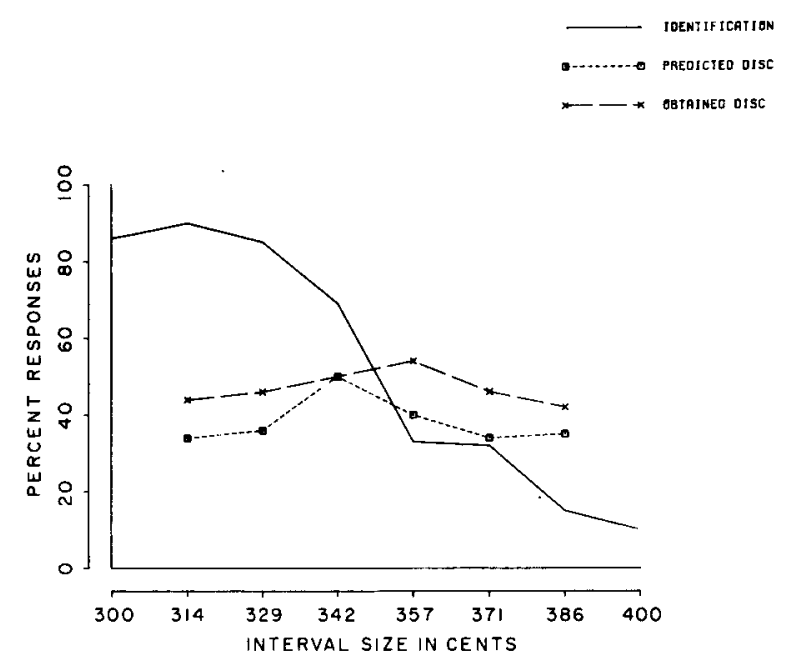

Figure 3. Mean percent identification responses for eight nonmusicians from Experiment 1, and mean percent correct predicted and obtained discrimination scores in the same subjects. 
and the peak of the predicted function did not correspond with the obtained peak. When discrimination performance of the musicians and nonmusicians was compared, it was found that the musicians scored much higher $[F(1,14)=23.0, p<.001]$, but there was no significant Subject Group by Stimulus Pair interaction, i.e., both groups discriminated between category pairs better than within categories. Nevertheless, the two functions differ in that the discrimination peak is sharper for the musicians, as shown by a comparison of the highest between-category to the lowest within-category scores for the two groups $[\mathrm{t}(14)=1.87, \mathrm{p}<.05]$. We should emphasize that there were wide individual differences here: most nonmusicians showed a flat discrimination function, while others increased their performance noticeably at category boundaries.

There were also interesting individual differences among musicians; data for two of the musicians are shown in Figures 4 and 5. Figure 4 shows data for the subject whose performance was the most categorical. Figure 5 shows data for a subject whose discrimination performance was near perfect at the major end of the continuum. This subject's performance is an extreme example of a trend present in most musicians (see Figure 2) for better discrimination at the major end than at the minor end. Of the eight musicians, three showed a drop in discrimination performance of $10 \%$ or less at the major end of the continuum. Moreover, the major category was found to be narrower than the minor category for almost all subjects in identification (Figure 1); i.e., musicians have stricter criteria for accepting a major third than a minor third. These facts may be due either to a learned narrower tuning of the major third, or to some physical characteristic of the major third [e.g., its place in the harmonic series, or that the major third approximates the bandwidth of a critical band in hearing (Scharf, 1970)].
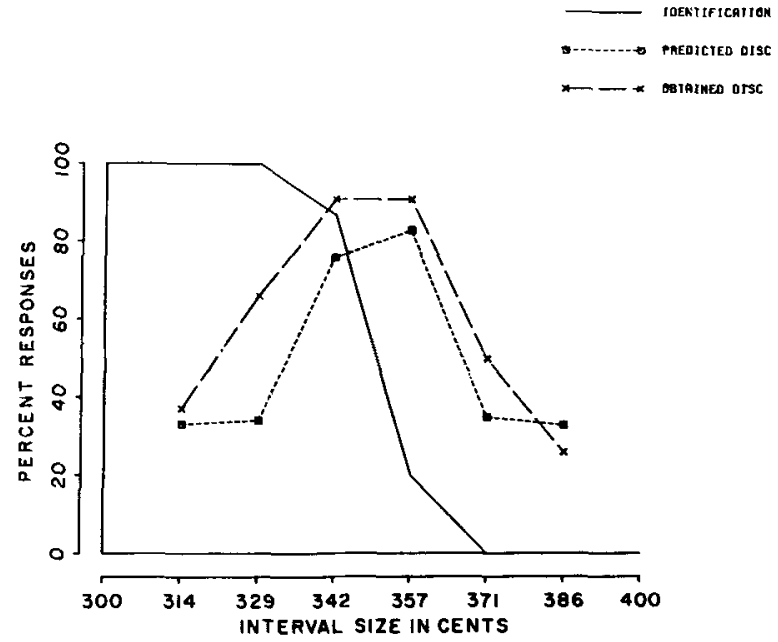

Figure 4. Individual data for one musician from Experiment 1: identification responses and percent correct predicted and obtained discrimination scores.

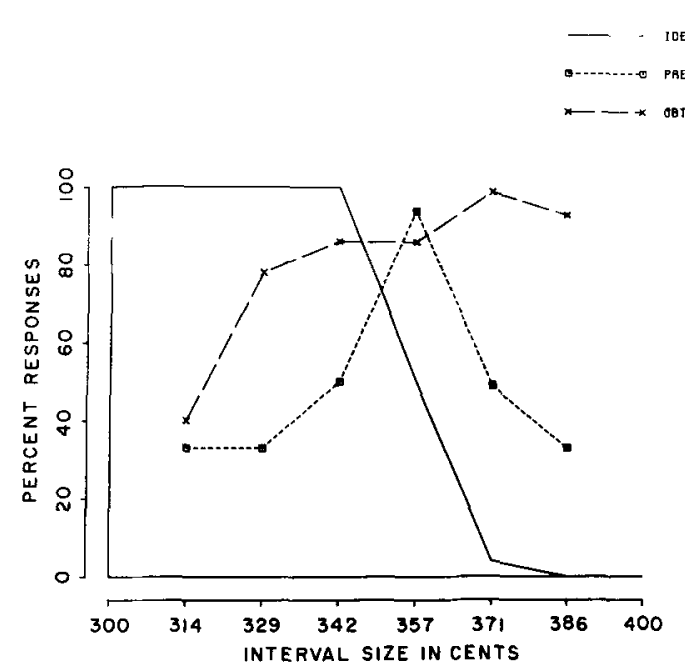

Figure 5. Individual data for one musician from Experiment I: identification responses and percent correct predicted and obtained discrimination scores.

It can be tentatively concluded that:

(1) Musicians can hear all distinctions better than nonmusicians, at least simply on an auditory basis. Otherwise, discrimination performance, at least within categories where musicians have no symbolic information to help them, would be equal for the two groups. The amount of auditory information available (on the order of $25 \%$ improvement over the predicted function) does seem to be greater than typically found in speech experiments, perhaps because of the extreme simplicity of the steady-state stimuli used here. This added information seems to be constant for all stimulus pairs. ${ }^{4}$

(2) Maximum difference in performance between the two groups is in the between-category judgments. In addition to a fairly good auditory basis for telling the pairs apart, musicians increase their performance drastically when labeling or symbolic distinctions become available to them. In physical terms, the distance between each discriminated pair is equal; only the additional information provided by categorical perception of the intervals could affect discrimination in such a manner.

(3) Nonmusicians also did slightly better discriminating across category boundaries than between them (Figure 3). However, this peak was not well predicted by the identification function. Two of the nonmusicians showed particularly marked peaks in discrimination at the category boundary. These subjects were about 10 years older than the other nonmusicians and also reported listening to music frequently. As a consequence, they may be in the process of acquiring categories similar to those possessed by the musicians through formal instruction.

(4) The reaction time data described above demonstrate operation of an auditory processing level, reflected in the changes in reaction time at the boundaries for the two tasks. 


\section{EXPERIMENT 2}

Experiment 1 demonstrated categorical-like perception by musicians for two-note intervals where the bottom note remained constant, and the frequency ratios were changed by varying only the frequency of the top note. Experiment 2 utilized major and minor thirds where the bottom as well as the top note varied; since intervals are defined by the frequency ratio between component tones, changing the frequency of the tones does not alter the interval. It was possible that in Experiment 1 subjects were basing the discrimination in part on the top note differences (in addition to interval size), thereby inflating performance. Thus, by randomizing the absolute position of the interval, subjects would be forced to listen to the interval information only.

\section{Method}

Subjects. The subjects were six Brown University undergraduate music students. All had had at least 14 years' experience in playing at least one instrument. They had all had at least one collegelevel music course which included ear training, and all were currently active in music.

Stimulus materials. The stimuli were similar to those in Experiment 1 , except that two more major-minor continua were constructed: one whose bottom note was always $F$ sharp $(370 \mathrm{~Hz})$ and whose top notes varied from $A(440 \mathrm{~Hz})$ to A sharp $(466 \mathrm{~Hz})$ in seven logarithmically equal steps, and another whose bottom note was always $G(392 \mathrm{~Hz})$ and whose top notes varied from B flat $(466 \mathrm{~Hz})$ to B natural $(493 \mathrm{~Hz})$. Each continuum thus had eight stimuli, the top note varying in 14.29 -cent steps, but always spanning the intervals from 300 to 400 cents. All other aspects of the stimuli were as in Experiment 1. Two identification tapes were constructed by presenting 5 repetitions of each of the 24 stimuli in two different random orders, thus making a total of 120 per tape. Other details of the stimuli were as in Experiment 1.

The discrimination tape was prepared, again by pairing members of a continuum two steps away to form oddity triads; however, each stimulus in the triad came from a different continuum, one from each of the three continua described above. Therefore, in order to choose the correct response, the subject would have to listen for the interval size (i.e., frequency ratio) and ignore the absolute pitches of the stimuli. The members of the three continua appeared in each position of the triads equally often; in addition, the odd stimulus also appeared equally often in each position. Two random orders of 72 trials each were prepared; other details of presentation were as in Experiment 1.

Procedure. The tapes were played on a Crown SX 822 tape recorder channeled through an Ampex tape recorder and then through both channels of Grason-Stadler TDH-39 headphones.

The identification task was similar to Experiment 1 except that the subjects recorded their responses on answer sheets and were run in small groups. The discrimination task was also run in conditions similar to those of Experiment 1 . The subjects were told beforehand that the stimuli would differ in absolute pitch, and that they were to select the stimulus different in interval size from the other two presented (i.e., different in degree of major or minor). They were also allowed to hear all three continua in order before they started the experiment.

\section{Results and Discussion}

Five of the six subjects showed highly inconsistent labeling data and chance or near chance discrimination performance. Only one listener (the most highly trained musician in the group) showed any evidence of being able to perform the task. Most subjects seemed to be misled into responding "minor" to the lower-pitched intervals, regardless of the size of the interval. Even for the one subject who responded with some consistency, there was little evidence for categorical perception, since labeling data did not show well-defined categories (endpoints were not even at $100 \%$ identification) and discrimination was near chance.

Since the subjects used here were different from those in Experiment 1, we wanted to insure that the lack of consistency was not due to the selection of subjects. Therefore, the six people were run on the same tapes from Experiment 1. The results were almost identical to those presented in Figure 1, so task and not subject variables are implicated.

These results are somewhat surprising in view of Burns and Ward's (1978) findings of good performance with intervals randomized with respect to absolute pitch. Though our discrimination task is somewhat more difficult than Burns and Ward's, we expected at least the identification data to be consistent. The inability of our subjects to ignore the absolute pitch of the intervals suggests that simultaneous intervals are processed differently from sequential intervals. In one sense, these results are not completely unexpected, given the high degree of stimulus uncertainty in this task. Nevertheless, the difference between sequential and simultaneous intervals remains to be explained.

It is also apparent that there is a great deal of contextual dependence with these stimuli; i.e., the relative size of the interval cannot be judged independently of the absolute pitches of the component tones.

We had expected to demonstrate, in this experiment, a total dependence on the symbolic level (a more abstract level where interval information and not absolute pitches are processed) for the discrimination task, since the acoustic characteristics of each stimulus in the triad were different. We did not succeed, however, as this manipulation made the task essentially impossible to perform. Nevertheless, we believe that there exists a symbolic level because of the discrimination peaks at category boundaries. Our next step, therefore, was aimed at exploring the symbolic level by means of the selective adaptation paradigm.

\section{EXPERIMENT 3}

Selective adaptation in speech usually involves the repetition of a stimulus sound related either phonetically or acoustically to the test continuum. A shift in the pattern of perception after adaptation may indicate that the adapting sound has fatigued units at that locus of processing. If the boundary is thought of as the point at which two channels 
have similar activation levels, then a shift can be predicted when the activity of one unit is reduced through adaptation. The direction of the shift is always toward the adapted category, since there will be relatively more identifications of ambiguous stimuli as members of the unadapted category. This experiment used an adaptor which was both acoustically and symbolically related to the test series in order to determine if these basic adaptation results could be obtained for musical interval perception.

\section{Method}

Subjects. Four musicians from Experiment 1 and an additional undergraduate music student served as subjects.

Stimulus materials. Identification and discrimination tapes from Experiment 1 were used. In addition, tape loops were constructed containing repeated presentations of the endpoint major third stimulus (FA: 400 cents), each followed by 500 msec of silence.

Procedure. The apparatus was identical to that in Experiment 1; the adaptation loops were played on a Sony tape recorder which fed into the amplifier. Timing was by stopwatch and the subjects were run individually or in pairs.

The subjects first heard the continuum endpoints for practice, then an identification tape and a discrimination tape were presented as in Experiment 1. After a short break, the adapting loop was presented for $2 \mathrm{~min}$ (120 presentations of the adaptor) followed by 10 trials of either identification or discrimination. One minute of adaptation was interspersed after every 10 trials thereafter. A different random order was used for preadaptation testing for each task. Order of task presentation was counterbalanced across subjects.

\section{Results and Discussion}

Mean percent identification before and after adaptation are shown in Figure 6. It is clear that there is a consistent shift in the boundary towards the adapted category, similar to that reported in speech experiments. Boundaries were calculated by converting responses in the boundary region to $\mathrm{z}$ scores and then finding the $50 \%$ point by linear interpolation. The average boundary change, from 355.9 to 366.45 cents is significant by a correlated means one-tailed $t$ test $[\mathrm{t}(4)=2.21, \mathrm{p}<.05]$.

The discrimination data before and after adaptation are shown in Figure 7. Obtained performance was significantly better than predicted on the basis of identification responses, as in Experiment 1. This was true for preadaptation $[\mathrm{F}(1,4)=30.28, \mathrm{p}<.001]$ as well as for postadaptation $[F(1,4)=171.0$, $\mathrm{p}<.001]$. The peak in the discrimination also shifted after adaptation. There was no interaction of predicted by obtained functions either pre- or postadaptation, indicating a good fit of the two functions.

These data are very similar to those obtained in speech experiments using the selective adaptation paradigm (e.g., Eimas \& Corbit, 1973). That the adaptation effect is not simply a response bias is shown by the change in the discrimination functions. The discrimination task does not depend explicitly on relative judgments of the category to which members of the oddity triad belong. The change in discrimination response after adaptation, therefore, more likely

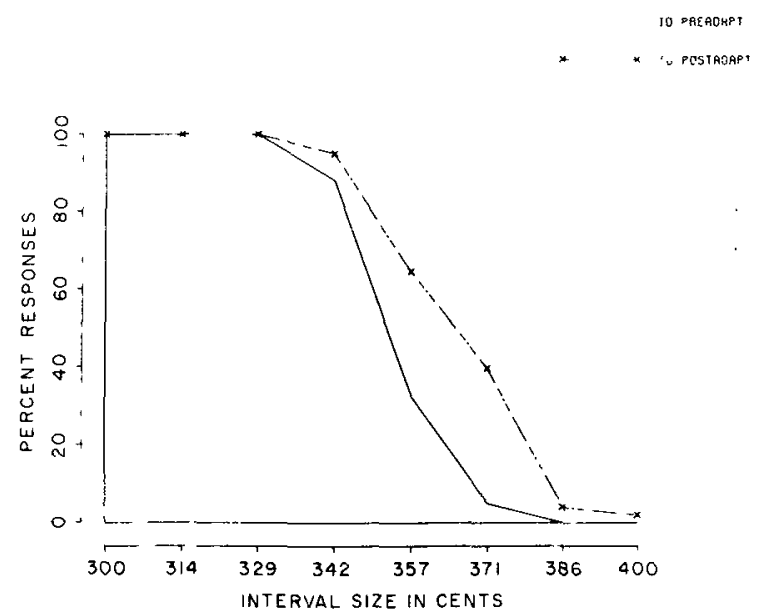

Figure 6. Mean percent identification responses before and after adaptation with major third ( 400 cent) stimulus for five musicians from Experiment 3.

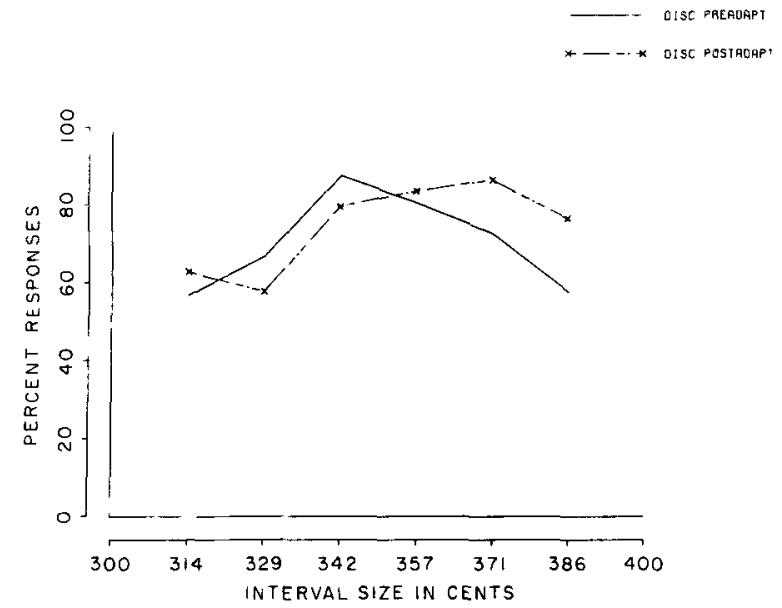

Figure 7. Mean percent obtained discrimination scores before and after adaptation with major third ( 400 cent) stimulus for five musicians from Experiment 3.

represents a true change in perceptual categories, as opposed to response bias.

Although the results from many selective adaptation experiments have been used as evidence for feature detector theories of perception, parsimony does not permit any similar formulation in the case of musical intervals. Neither do we believe that the effect takes place at a level of response organization. ${ }^{5}$ Instead, it seems likely that we are affecting the output of some channel of analysis. We do not know from these data the locus of the adaptation effect, nor can we describe the stimulus characteristics to which the adapted channel is sensitive. The most obvious way to investigate these questions is by varying the adaptor and measuring the adaptation effect. This was undertaken in the next experiment.

\section{EXPERIMENT 4}

An important question raised by Experiment 3 is 
whether the adapted channels are responding to interval information independently from absolute frequency. To answer this question, a set of adaptors was constructed in which the pitches were different from the test continuum. Adaptors where the pitches were the same (as in Experiment 3) were also used. Naturally, the intervals were always major or minor thirds (endpoints of continua), thus maintaining the interval information constant. In addition, single note adaptors were used to see if the adapted channel was sensitive to a single tone, as opposed to an interval. Finally, interaural transfer of the adaptation effect was tested to determine the locus of the effect.

\section{Method}

Subjects. The subjects from Experiment 2 were used, along with an additional five musicians from the Providence area who met the criteria stated above.

Stimulus materials. The identification tapes from Experiment 1 (FA-FA flat continuum) were used as the test stimuli in all conditions. In Conditions 1-6, adaptors were always endpoint stimuli from one of two continua: FA (major third low pitch), FA flat (minor third low pitch), GB (major third high pitch), and GB flat (minor third high pitch). In Conditions 7 and 8 , the adaptor was the single tone A flat $(415 \mathrm{~Hz})$, which is the top note of the lowpitch minor interval. Other details of the stimuli were as in Experiment 3.

Procedure. The apparatus used was identical to that in Experiment 2. Adapting stimuli were played on the Ampex tape recorder, test stimuli were played on the Crown.

On each day, the subject was given a preadaptation identification test followed by an adaptation run (see Experiment 3 for details of adaptation presentation). Only one adaptor (i.e., one condition) per day was used. In Conditions 1 and 2 , the minor adaptors (FA flat and GB flat) were used. Since the test stimuli were taken from the low-pitch (FA to FA flat) continuum, Condition 1 constitutes a replication of the identification task of Experiment 3. The GB flat interval is the same frequency ratio ( 300 cents) as FA flat, but it is transposed upward by a full tone (200 cents), so neither of the individual tones is the same as the tones in the test series. Conditions 3 and 4 used the same adaptors as Conditions 1 and 2 , but the adapting stimuli were presented monaurally, left ear only for all subjects, and the test stimuli always to the right ear. Preadaptation tests were also done monaurally (right ear only) for these conditions. Conditions 5 and 6 used the two major ( 400 -cent) adaptors (FA and GB) from both low- and high-pitch continua, i.e., the opposite endpoints from the adaptors used in Conditions 1 and 2. Conditions 7 and 8 used the single tone A flat as the adaptor; in Condition 7, presentation was binaural; in Condition 8 , the adaptor was presented to the left ear, the test series to the right ear. Order of presentation was counterbalanced across subjects for each pair of conditions.

\section{Results and Discussion}

Boundaries before and after adaptation were determined as in Experiment 3. The amount of shift (preadaptation boundary minus postadaptation boundary) for each condition is shown in Table 1 . In spite of considerable between-subject variation, the shifts were in the predicted direction for all subjects in the conditions where the adaptor came from the same frequency continuum as the test series (Conditions 1, 3 , and 5). Note that the shifts were approximately of equal size for each endpoint adaptor (i.e., major and minor adaptors had equally strong effects). There was no significant effect when the adaptor preserved the proper frequency ratio but was pitched higher than the test continuum (Conditions 2, 4, and 6). In addition, when adaptor and test were presented to different ears (i.e., comparing Conditions 1 and 3), the amount of shift was statistically unchanged with respect to binaural presentation. Finally, when the top note of the low minor interval (A flat) was presented alone, the adaptation effect was just as great as when the entire interval was presented. This effect, too, showed $100 \%$ interaural transfer. ${ }^{6}$

The fact that there was no significant effect in the conditions where the adaptors shared the appropriate frequency ratio with the test continuum but not the absolute pitches (Conditions 2, 4, and 6) is quite important. This finding implies that the symbolic level of analysis (i.e., where the decision major or minor is made) is not being tapped by the adaptation technique. Moreover, it implies that whatever is being adapted is frequency specific. The result from the single-note condition (7) indicates that the adapted level is sensitive to single tones and not to ratios. Perhaps the adapted level is where single-tone extraction takes place, before the ratio is computed.

It was also found that $100 \%$ interaural transfer occurred (in Conditions 3 and 8). This result implies that the effect is operating centrally. Since transfer was also obtained in the single-note condition (8), it seems safe to conclude that the effect of the inter-

Table 1

Amount of Boundary Shift, in Cents, for Various Adapting Conditions in Experiment 4

\begin{tabular}{|c|c|c|c|c|c|c|c|c|}
\hline \multirow[b]{2}{*}{ Subject } & \multicolumn{8}{|c|}{ Condition } \\
\hline & $1\left(\mathbf{F A}^{b}\right)$ & $2\left(\mathrm{~GB}^{b}\right)$ & $3\left(\mathrm{FA}^{b}\right)$ & $4\left(G^{b}\right)$ & $5(\mathrm{FA})$ & $6(\mathrm{~GB})$ & $7\left(\mathrm{~A}^{b}\right)$ & $8\left(A^{b}\right)$ \\
\hline $\begin{array}{l}\text { L.M. } \\
\text { M.S. } \\
\text { L.N.* } \\
\text { J.B. } \\
\text { K.F. } \\
\text { M.R. }\end{array}$ & $\begin{array}{r}8.86 \\
7.28 \\
5.55 \\
4.29 \\
9.57 \\
.14\end{array}$ & $\begin{array}{r}6.43 \\
6.29 \\
-4.57 \\
1.00 \\
10.00 \\
-3.14\end{array}$ & $\begin{array}{l}8.00 \\
3.00 \\
5.00 \\
8.28 \\
4.86 \\
1.28\end{array}$ & $\begin{array}{r}10.86 \\
3.00 \\
-1.00 \\
.57 \\
-1.29 \\
-4.14\end{array}$ & $\begin{array}{r}-13.70 \\
-2.86 \\
-4.57 \\
-2.57 \\
-11.00\end{array}$ & $\begin{array}{r}3.43 \\
2.14 \\
-\quad .71 \\
-3.00 \\
-3.00\end{array}$ & $\begin{array}{r}5.55 \\
11.85 \\
\\
8.00 \\
3.14 \\
12.57\end{array}$ & $\begin{array}{r}12.14 \\
7.14 \\
\\
10.00 \\
-3.57 \\
.57\end{array}$ \\
\hline $\begin{array}{l}\text { Mean } \\
p<\end{array}$ & $\begin{array}{l}5.95 \\
.005\end{array}$ & $\begin{array}{l}2.67 \\
\text { n.s. }\end{array}$ & $\begin{array}{l}5.07 \\
.005\end{array}$ & $\begin{array}{l}1.33 \\
\text { n.s. }\end{array}$ & $\begin{array}{r}6.94 \\
.025\end{array}$ & $\begin{array}{r}-.23 \\
\text { n.s. }\end{array}$ & $\begin{array}{r}8.22 \\
.01\end{array}$ & $\begin{array}{l}5.26 \\
.10 \dagger\end{array}$ \\
\hline
\end{tabular}

Note-Adaptor and test were presented to separate ears in Conditions 3, 4, and 8. "This subject failed to complete all conditions. tSee text, Footnote 4. 
val adaptors (Conditions 1, 3, and 5) can be most parsimoniously accounted for by the presence of the top note in the adaptor. ${ }^{7}$

\section{GENERAL DISCUSSION}

These experiments have shown that a phenomenon similar to categorical perception of speech sounds exists for perception of simultaneous musical intervals. That is to say, trained musicians have internalized the concepts of major and minor third to such an extent that sounds they can label differently are more discriminable than sounds given the same name. But, while Burns and Ward (1978), Siegel and Siegel (1977a, 1977b), and Blechner (Note 2) have virtually duplicated with musical stimuli results found for the perception of consonants, in this study we found limits to the categoricalness of perception, at least for simultaneous major and minor thirds.

There is an a priori reason to expect categorical perception of musical intervals by musicians. An interval, like a speech sound, conveys invariant information despite being made up of varying physical signals. For example, in a major third, the higher note is placed into a frequency ratio of $1.26: 1$ with the bottom note (i.e., 400 cents), regardless of the absolute frequency of the individual notes. In Western music theory, frequency ratios perform the same harmonic and melodic function in any key (i.e., without regard to any specified absolute frequency).

In agreement with this argument, Siegel and Siegel (1977b) found consistent performance on a magnitude estimation task and a labeling task using successive intervals, even when the bottom note of the intervals was varied among the seven diatonic tones from the C major scale. Burns and Ward (1978) constructed intervals whose absolute frequency varied within a range of 360 cents, and found categorical perception with consistent labeling and discrimination performance no higher than predicted on the basis of identification. There is even evidence that interval information is stored in memory as an abstract entity (not as particular notes) from the interference data gathered by Deutsch (1978; Deutsch $\&$ Roll, 1974). These studies reinforce the idea that the theoretical invariance of the interval (in music) extends to perceptual invariance, as is the case with acoustically different, but perceptually identical, consonantal speech sounds. However, using simultaneous intervals in tasks very similar to those used by Burns and Ward, we found, in Experiment 2, that our musicians were unable to successfully apply the concept of major and minor thirds when absolute pitch was randomized.

Moreover, these results were confirmed in Experiment 4 , where adaptation by an interval which shared symbolic, but not acoustic, similarities with the test series failed to shift the category boundary. We must, then, conclude that there is a fundamental difference between processing successive and simultaneous intervals. In simultaneous intervals, listeners must first isolate the component tones and then analyze their frequency relationship. This perceptual isolation is accomplished in successive intervals by the temporal separation between component tones. Categorical perception can only be employed by the listener if this frequency ratio has been abstracted. Perhaps the perceptual complexity of simultaneous intervals forces analysis to occur only at a frequencyspecific level, especially in the rigorous tasks we presented to the subjects.

Most contemporary models of pitch perception (Goldstein, 1973; Wightman, 1973a, 1973b) involve a two-stage mechanism which is relevant to some of our results. In the first stage, the incoming complex acoustic waveform is subjected to a Fourier transform which computes the spectral characteristics of the signal. From this, the individual components of the complex could be identified. This stage would naturally be frequency specific. The second stage is typically viewed as a pattern recognition or template match that searches out the best fitting fundamental for the given harmonics. In the case of musical intervals, subjects are not listening for a periodicity pitch arising from the two tones, but rather to the interval information; it is therefore reasonable to propose that the output of the pitch extractor could feed into a third stage that computes ratios.$^{8}$ Thus, discrimination peaks and reaction time increases at category boundaries, such as were found in our first experiment, could perhaps arise from the operation of this third stage, since it is here that particular interval information would be stored. This stage would most likely be set up to accept a range of particular ratios as exemplars of prototype intervals on the basis of learning, and thus would be associated with longterm memory storage. We cannot completely rule out the possibility, however, that there could exist some natural propensity of the auditory system on which the tuning for the learned intervals would be superimposed (e.g., a propensity for small-integer ratios).

Only individual note information (i.e., pitches of the components) would be stored at the pitch extraction stages. The availability of this auditory, analog information would explain the higher than predicted discrimination performance in Experiment 1, because the differences between the top notes of the intervals could be compared to each other. This scheme would also explain the adaptation effects. Since single-note adaptors were effective, we must assume that adaptation fatigues the single-note analyzers. Further evidence that is consistent with this model comes from the fact that our adaptation effects all appear to be centrally mediated, as do the effects associated with complex tone perception (Houtsma \& Goldstein, 1972). 
Blechner (Note 2) also reported higher than predicted discrimination for some of his musicians in tasks very similar to ours. It is interesting that he found that when subjects were given only a singlenote continuum (our top note), their discrimination functions did not show the peak characteristic of categorical perception. It seems clear, then, that although the auditory, noncategorical information may increase discrimination performance, it cannot be the sole basis for it.

As for the results of the second experiment, which used intervals randomized with respect to absolute frequency, it seems that the task did not successfully engage the ratio analysis stage. Rather, the system may have been overloaded at some point (too many stimuli, too short presentation durations, etc.), and most subjects fell back on using a simpler (and incorrect) strategy of relying on the absolute frequency of the stimuli.

The model outlined here bears a strong resemblance to a model for interval recognition discussed by Deutsch $(1969,1975)$. In her formulation, there is a primary array where individual note information is stored (e.g., F or A). This stage is connected to a specific interval stage, where interval information at specific pitches is stored (e.g., FA or GB flat). The information from this second stage then converges onto a third, where abstract (i.e., frequency-independent) interval information is processed. Clearly, this model is in basic agreement with our data. Deutsch's model offers another explanation of the data from Experiment 2 . If that paradigm only tapped a frequencyspecific interval stage, the subjects would have been unable to successfully compare stimuli from different continua, as these would have been coded according to interval size and absolute frequency, rather than the abstract interval. In other words, a true major third whose bottom note was $\mathrm{F}$ would not have been coded as equivalent to one whose bottom note was $\mathrm{G}$.

Another effect which we found here that has not been reported by other studies in this field was the generally better discrimination (in Experiment 1) of stimuli from the major end of the continuum than from the minor end. This effect is probably also related to the narrower category in identification for major stimuli in Experiment 1. As mentioned above, this special nature of the major third may be due to some special acoustical characteristics of this particular interval, or it may be due to the major third having a special place in the Western musical system. One point which would argue against the former interpretation is that the better within-category discrimination of major than of minor intervals was completely absent for the nonmusicians (Figure 3 ).

We wish now to bring up the question of how relevant these findings are for the perception of real music. Neither tasks nor stimuli used here resemble those normally found in music. Even so, it stands to reason that categorical perception would be even more advantageous in the concert hall than in the laboratory. Ward (1970) has cited data showing that in real performances there is much variance in the frequency of the notes being played, but that it is not usually noticed by listeners. Categorical perception results when a listener ignores small pitch irregularities and thereby pays more attention to more important aspects of the music. However, a truly out of tune performance is obviously detectable to most listeners. This phenomenon shows up in the laboratory in the appearance of the auditory component (i.e., higher than predicted results) in the discrimination task. Also, recall that in Experiment 1 we found uniformly good performance even within categories for the discrimination task with three steps (43 cents) between stimuli.

Further, the principal result from Experiment 2, i.e., that intervals could not be judged consistently when the absolute frequency of the component tones was varied, could be interpreted in terms of real-life musical systems as one reason for the establishment of such devices as key centers and tonalities. A key center provides a framework for tones in a scale such that some tones are more important than others; chords built on these tones gravitate away from and back to these more important tones (e.g., in a major diatonic scale, the harmonic roots of chords move towards the first, third, and fifth scale degrees). Thus, the tonal centers provide a context for the interpretation of individual harmonic intervals, without which the intervals might not be judged correctly. We have no doubt that in real music there are many such higher order constraints on processing the basic acoustic information, perhaps analogous to the syntactic and semantic constraints that apply to language.

The model that we have discussed obviously remains preliminary and somewhat vague. There remain many issues to be explained; specificially, a precise description of the mechanism underlying the adaptation effect has not been given. Also, sufficient data to distinguish between a frequency-specific interval stage and an abstract interval stage is lacking, and an adequate description of the characteristics of these higher order stages has not yet been given. Finally, the issue of how experience affects categorical perception is unclear-it is not known if training results in the phenomena we have seen, or if there are natural differences between listeners such that those who process musical stimuli in a certain way are also those who seek out training and thus become musicians.

\section{REFERENCE NOTES}

1. Fujisaki, H., \& Kawashima, T. Some experiments on speech perception and a model for the perceptual mechanism. Annual Report of the Engineering Research Institute, University of Tokyo, 1970, 29, 207-214. 
2. Blechner, M. J. Musical skill and the categorical perception of harmonic mode. Haskins Laboratories Status Report on Speech Perception, 1977, SR-51/52, 139-174.

\section{REFERENCES}

Burns, E. M., Carney, A. E., \& Ward, W. D. Balancing the scales for music and speech. Journal of the Acoustical Society of America, 1976, 60, S94(A).

Burns, E. M., \& W ARD, W. D. Categorical perception-phenomenon or epiphenomenon: Evidence from experiments in the perception of melodic musical intervals. Journal of the Acoustical Society of America, 1978, 63, 456-468.

Cooper, F. S., \& Mattingly, I. G. A computer-controlled PCM system for the investigation of dichotic speech perception. Journal of the Acoustical Society of America, 1969, 46, $115(\mathrm{~A})$.

Cutting, J. E., \& Rosner, B. S. Categories and boundaries in speech and music. Perception \& Psychophysics, 1974, 16, 564-570.

Cutting, J. E., Rosner, B. S., \& Foard, C. F. Perceptual categories for musiclike sounds: Implications for theories of speech perception. Quarterly Journal of Experimental Psychology, 1976, 28, 361-378.

Deutsch, D. Music recognition. Psychological Review, 1969, 76, 300-307.

DEUTSCH, D. The organization of short-term memory for a single acoustic attribute. In D. Deutsch \& J. A. Deutsch (Eds.), Shortterm memory. New York: Academic Press, 1975.

Deutsch, D. Interactive effects in memory for harmonic intervals. Perception \& Psychophysics, 1978, 24, 7-10.

Deutsch, D., \& Role, P. L. Error patterns in delayed pitch comparisons as a function of relational context. Journal of Experimental Psychology, 1974, 103, 1027-1034.

DienL, R. L. The effect of selective adaptation on the identification of speech sounds. Perception \& Psychophysics, 1975, 17, 48-52.

Diehl, R. L., Elman, J. L., \& McCusker, S. B. Contrast effects on stop consonant identification. Journal of Experimental Psychology: Human Perception and Performance, 1978, 4, 599-609.

Ermas, P. D. The relation between identification and discrimination along speech and nonspeech continua. Language \& Speech, $1963,6,206-217$.

Eimas, P. D., \& Corbit, J. D. Selective adaptation of linguistic feature detectors. Cognitive Psychology, 1973, 4, 99-109.

Eimas, P. D., Cooper, W. E., \& Corbit, J. D. Some properties of linguistic feature detectors. Perception \& Psychophysics, $1973,13,247-252$.

Eimas, P. D., \& Miller, J. L. Effects of selective adaptation on the perception of speech and visual patterns: Evidence for feature detectors. In H. L. Pick \& R. D. Walk (Eds.), Perception and experience. New York: Plenum, 1978.

Goldstein, J. L. An optimum processor theory for the central formation of the pitch of complex tones. Journal of the Acoustical Society of America, 1973, 54, 1496-1516.

Houtsma, A. J. M., \& Goldstein, J. L. The central origin of the pitch of complex tones. Journal of the Acoustical Society of America, 1972, 51, 520-529.

JesteaD, W., \& BilgeR, R. C. Intensity and frequency discrimination I: One- and two-interval paradigms. Journal of the Acoustical Society of America, 1974, 55, 1266-1279.

Liberman, A. L., Harris, K. S., Hoffman, H. S., \& Griffirh, B. S. The discrimination of speech sounds within and across phoneme boundaries. Journal of Experimental Psychology, $1957,54,358-368$.

Liberman, A. L., Harris, K. S., Kinney, J. A., \& Lane, H. The discrimination of relative onset time of the components of certain speech and nonspeech patterns. Journal of Experimental Psychology, 1961, 61, 379-388.
LOCKe, S., \& Kellar, L. Categorical perception in a nonlinguistic mode. Cortex, 1973, 9, 355-369.

Macmillan, N. A., Kaplan, H. L., \& Creelman, C. D. The psychophysics of categorical perception. Psychological Review, $1977,84,452-471$.

Mattingly, I. G., Liberman, A. L., Syrdal, A., \& Halwes, T. Discrimination in speech and nonspeech modes. Cognitive Psychology, 1971, 2, 131-157.

Miller, G. A. The magical number seven plus or minus two: Some limits on our capacity for processing information. Psychological Review, 1956, 63, 81-97.

Miller, J. D., Wier, C. C., Pastore, R. E., Kelly, W. J. \& Dooling, R. J. Discrimination and labeling of noise-buzz sequences with varying noise-lead times. Journal of the Acoustical Society of America, 1976, 60, 410-417.

PAstore, R. E. Categorical perception: A critical reevaluation. In S. K. Hirsh, D. H. Eldredge, I. J. Hirsh, \& S. R. Silverman (Eds.), Hearing and Davis: Essays honoring Hallowell Davis. St. Louis, Mo: Washington University Press, 1976.

Pastore, R. E., Ahroon, W. A., Baffuto, K. J., Freidman, C., Puleo, J. S., \& Fink, E. A. Common factor model of categorical perception. Journal of Experimental Psychology: Human Perception and Performance, 1977, 3, 686-696.

Pison I, D. B. Auditory and phonetic memory codes in the discrimination of consonants and vowels. Perception \& Psychophysics, 1973, 13, 253-260.

Pisoni, D. B. Auditory short term memory and vowel perception. Memory \& Cognition, 1975, 3, 7-18.

Pisoni, D. B. Identification and discrimination of the relative onset time of two component tones: Implications for voicing perception in stops. Journal of the Acoustical Society of America, 1977, 61, 1352-1361.

PIsONI, D. B., \& TASH, J. Reaction times to comparisons within and across phonetic categories. Perception \& Psychophysics, $1974,15,285-290$.

RAZ, I., \& BRANDT, J. F. Categorical perception of nonspeech stimuli by musicians and nonmusicians. Journal of the Acoustical Society of America, 1977, 62, S60(A).

SAwusch, J. R. Peripheral and central processes in selective adaptation of place of articulation in stop consonants. Journal of the Acoustical Society of America, 1977, 62, 738-750.

ScharF, B. Critical bands. In J. V. Tobias (Ed.), Foundations of modern auditory theory (Vol. 1). New York: Academic Press, 1970.

Shower, E. G., \& Biddulph, R. Differential pitch sensitivity of the ear. Journal of the Acoustical Society of America, 1931, $3,275-277$

Siegel, J. A., \& Siegel, W. Absolute identification of notes and intervals by musicians. Perception \& Psychophysics, 1977, 21, 143-152. (a)

Siegel, J. A., \& Siegel, W. Categorical perception of tonal intervals: Musicians can't tell sharp from flat. Perception \& Psychophysics, 1977, 21, 399-407. (b)

Simon, H. J., \& Studdert-Kennedy, M. Selective anchoring and adaptation of phonetic and nonphonetic continua. Journal of the Acoustical Society of America, 1978, 64, 1338-1357.

Studdert-Kennedy, M., Liberman, A. M., Harris, K. S., \& COOPER, F. S. Motor theory of speech perception: A reply to Lane's critical review. Psychological Review, 1970, 77, 234-249.

Studdert-Kennedy, M., Liberman, A. M., \& Stevens, K. N Reaction time to synthetic stop consonants and vowels at phoneme centers and at phoneme boundaries. Journal of the Acoustical Society of America, 1963, 35, 1900(A).

W ARD, W. D. Musical perception. In J. V. Tobias (Ed.), Foundations of modern auditory theory (Vol. 1). New York: Academic Press, 1970.

Wightman, F. L. Pitch and stimulus fine structure. Journal of the Acoustical Society of America, 1973, 54, 397-406. (a)

Wightman, F. L. The pattern-transformation model of pitch Journal of the Acoustical Society of America, 1973, 54. 407-416. (b) 


\section{NOTES}

1. By peripheral and central, we simply mean processing stages before and after information from the two ears is combined, respectively.

2. The difference threshold for frequency discrimination varies considerably depending on the methods used, loudness and frequency of the tones, etc., but within the frequency range we are using here, the DL is typically on the order of $2-4 \mathrm{~Hz}$ (Jestead \& Bilger, 1974; Shower \& Biddulph, 1931). Thus, we are working well above threshold.

3. The formula we are using is commonly used in speech research:

$$
P(\text { corr })=(1 / 3)\left[1+2\left(\mathrm{P} 1-\mathrm{P} 1^{\prime}\right)^{2}\right],
$$

where $\mathrm{P} 1$ is the probability of assigning one member of a pair to a category, and $\mathrm{Pl}^{\prime}$ is the probability of assigning the other member to that category.

4. This claim must be made very cautiously since we have no unbiased estimate of discriminatory sensitivity for each stimulus comparison (cf. Macmillan, Kaplan, \& Creelman, 1977).

5. Recent accounts of adaptation experiments have emphasized sensory contrast and anchoring rather than sensory fatigue (Diehl, Elman, \& McCusker, 1978; Simon \& Studdert-Kennedy, 1978). We cannot at present rule out such influences in our results. It is interesting that Siegel and Siegel (1977a), using sequential intervals with musicians, failed to find any contrast effects. It will be interesting to see if the same holds true for simultaneous intervals.

6. This effect did not achieve significance with the original five subjects; however, it seemed to be mainly due to a single deviant score, that of a subject who later admitted not paying attention during the task. To be certain that there was interaural transfer in this condition, five new subjects were run in Conditions 7 and 8 of Experiment 4. The results showed significant adaptation in both conditions in the predicted direction.

7. This might also explain the lack of effect in the conditions where the adaptor did not come from the same continuum as the test series. In Condition 2, for example, the adaptor is GB flat; the critical tones in the test series go from $A$ to $A$ flat, and since B flat is 100 cents above $A$ and $G$ is 100 cents below A flat, it is possible that they had a mutually cancelling effect. At this point, however, we do not know the tuning functions associated with the adaptation effect, so we cannot be certain that adaptation would result when a single tone adaptor is as much as 100 cents away from the endpoint of the test series.

8. If the two tones that make up the interval were harmonic complexes (with or without fundamentals) rather than sinusoids, then the tone extraction stage would be slightly more involved, as two different spectral pattern matches would have to be made in order to determine the two fundamentals. The particulars of this process are necessarily complicated, but they need not concern us here; the end result of this processing stage would still be two pitch percepts from which the musical interval could be computed. We assume, then, that the model described would be valid for complex tones as well as for pure tones, though we plan to test this assumption empirically in future work.

(Received for publication March 8, 1979; revision accepted August 17, 1979.) 